\title{
Vaginal vault dehiscence with evisceration after total laparoscopic hysterectomy
}

\author{
Rakesh Sinha Pratima Kadam • \\ Meenakshi Sundaram • Chaitali Mahajan • \\ Parul Shah • Smita Lakhotia • Gayatri Rao
}

Received: 2 April 2010 / Accepted: 9 June 2010 / Published online: 3 July 2010

(C) Springer-Verlag 2010

Keywords Total laparoscopic hysterectomy - Evisceration . Vault dehiscence

Vault dehiscence and evisceration of peritoneal contents is a rare complication following total hysterectomy. A 43-yearold lady presented with history of something soft coming out per vaginum. She underwent total laparoscopic hysterectomy 2 months back for 16-week fibroid uterus.

The surgery was uneventful, vaginal vault was sutured laparoscopically with no 1 polyglactin, interrupted figure of eight sutures. She was discharged after $48 \mathrm{~h}$ with uneventful recovery and subsequently presented with mass protruding per vaginum. Speculum examination showed loops of small bowel protruding in the vagina (Fig. 1). She was hospitalized and scheduled for reduction of the prolapsed bowel with vault repair.

On examination under anaesthesia, there was complete dehiscence of the vaginal vault with loops of small bowel protruding through the defect. To maintain the pneumoperitoneum vagina was blocked using a simple vaginal tampon soaked in betadine solution (Fig. 2) [1].

On Laparoscopic evaluation the entire vault was deficient. There were no bowel, bladder or omental adhesions to the vault. The prolapsed ileal loops were seen just at the vault, with healthy pink colour and regular peristalsis. On giving Trendelenburg's position the reposition was smooth. After confirming that the vault was free of any

R. Sinha $\cdot$ P. Kadam $(\varangle) \cdot$ M. Sundaram $\cdot$ C. Mahajan $\cdot$ P. Shah S. Lakhotia $\cdot$ G. Rao

Beams Specialty Hospital for Women,

Mumbai, India

e-mail: pratimakadam77@hotmail.com adhesions, the vault was closed vaginally using interrupted no 1 Polydioxone II sutures (Fig. 3). Patient was discharged on the third post-operative day and is asymptomatic at 6-month follow-up.

Vaginal vault dehiscence with evisceration posthysterectomy can occur up to few weeks to even few years after hysterectomy. The commonest organ to eviscerate is small bowel, particularly terminal ileum. Several factors which may contribute to weakness of vaginal apex are poor surgical techniques, post-operative wound haematoma and cuff infection, post-menopausal status, any activity resulting in increased intra-abdominal pressure, early resumption of sexual activity, chronic steroid administration, history of previous radiotherapy in cases of radical hysterectomy [2] and systemic illness like diabetes mellitus and immunecompromised status [3].

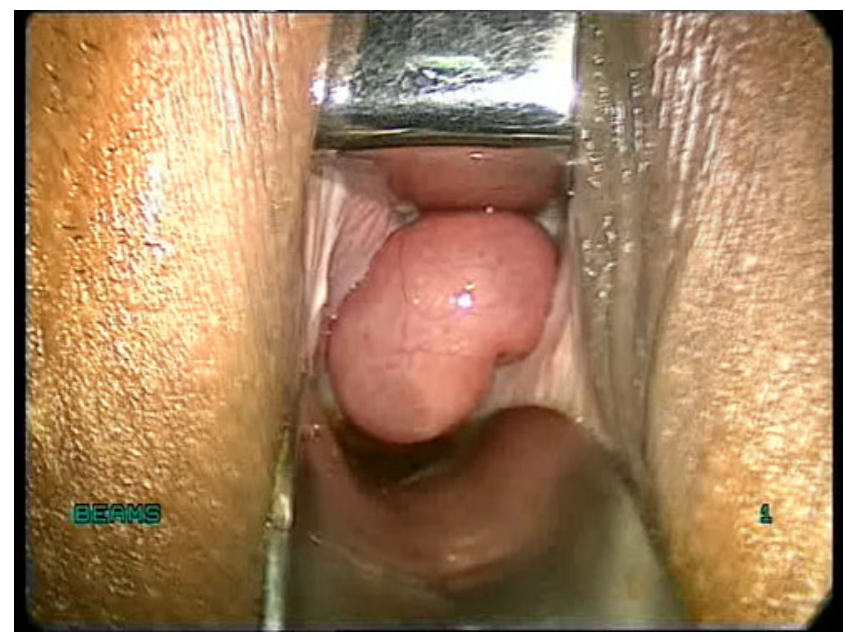

Fig. 1 Small bowel protruding through the vault; $112 \times 84 \mathrm{~mm}(300 \times$ 300 DPI) 


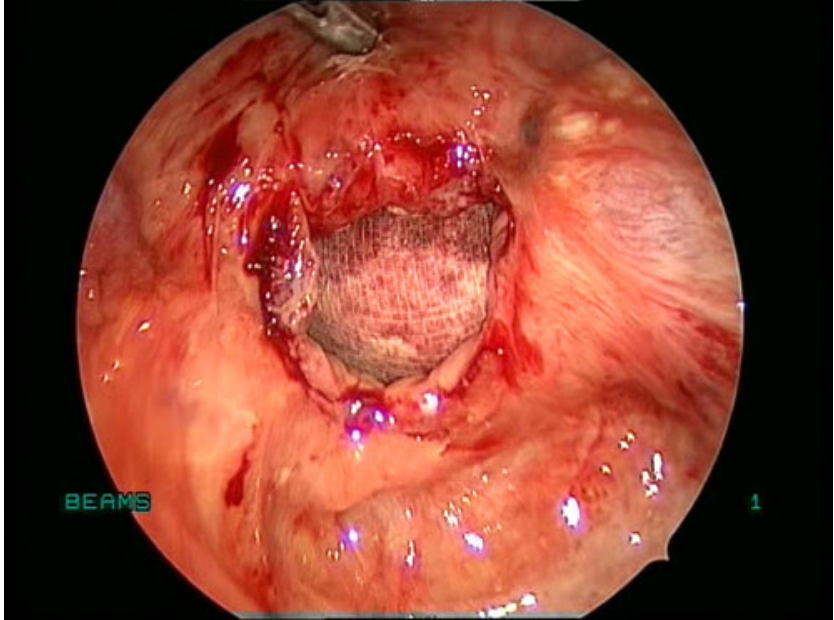

Fig. 2 Complete vault dehiscence viewed laparoscopically with vaginal tampon; $112 \times 84 \mathrm{~mm}(300 \times 300 \mathrm{DPI})$

\section{References}

1. Rakesh Sinha, Meenakshi Sundaram, Smita Lakhotia et al (2009) Total Laparoscopic hysterectomy for large uteri. J Gynec Endosc Surg 1(1):34-39 2. Somkuti SG, Vieta PA, Daugherty JF, Hartley LW, Blackmon EB (1994) Transvaginal evisceration after hysterectomy in premeno-

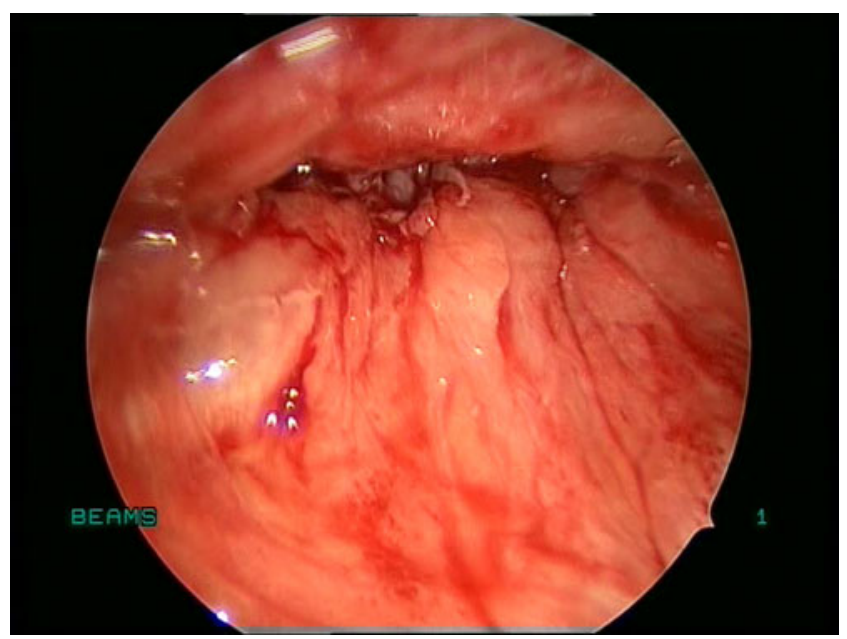

Fig. 3 Vaginal vault after suturing; 112x84mm $(300 \times 300 \mathrm{DPI})$

pausal women: a presentation of three cases. Am J of Obstet Gynecol 171:567-568

3. Agdi M, Al-Ghafri W, Antolin R, Arrington J, O'Kelley K, Thomson AJ, Tulandi T (2009) Vaginal Vault dehiscence after hysterectomy, J Minim Invasive Gynecol. Department of Obstetrics and Gynecology, McGill University, Montreal, Quebec, Canada, pp 313-317 